\title{
Steady State Computations for Wave Propagation Problems*
}

\author{
By Björn Engquist and Bertil Gustafsson
}

\begin{abstract}
The behavior of difference approximations of hyperbolic partial differential equations as time $t \rightarrow \infty$ is studied. The rate of convergence to steady state is analyzed theoretically and expe imentally for the advection equation and the linearized Euler equations. The choice of difference formulas and boundary conditions strongly influences the rate of convergence in practical steady state calculations. In particular it is shown that upwind difference methods and characteristic boundary conditions have very attractive convergence properties.
\end{abstract}

1. Introduction. For computing steady state solutions to problems in fluid mechanics, the time-dependent formulation is often used. There are several mechanisms that drive the solution to a steady state. In this paper we shall concentrate on the dissipation effect due to the boundary conditions, and not to the effect of friction and viscosity. Therefore we shall study hyperbolic partial differential equations where the boundary effects are dominant. The results are also valid for more general classes of differential equations of essentially hyperbolic character, as for example the Navier-Stokes equations for high Reynolds numbers.

The purpose of this paper is to analyze the convergence properties to steady state both for the continuous problem and the corresponding discrete approximation. The basis for this study is the behavior of the spectrum of the differential and the difference operators, respectively. Two model problems are considered, the scalar advection problem and the isentropic Euler equation problem. The latter problem is studied in a two-dimensional geometry corresponding to channel flow. It is shown that the choice of boundary conditions radically affects the convergence rate to steady state as time increases. The asymptotic rate may actually change from exponential to algebraic, and for certain sets of boundary conditions there may be no convergence at all.

The convergence rate for time-marching procedures has been discussed by others. For example, in [4] Giles investigated the eigenmodes of the solution of the one-dimensional Euler equations under various boundary conditions. Eriksson and Rizzi in [2] studied the convergence rate for a time-marching centered finite-volume

Received January 9, 1986; revised August 11, 1986.

1980 Mathematics Subject Classification (1985 Revision). Primary 65M10.

* This work was supported by NSF Grant No. DMS85-03294, ARO Grant No. DAAG29-85-K-0190, NASA Consortium Agreement No. NCA2-IR390-403 and by the Swedish Board for Technology, Research program for civil aircraft technology. 
method applied to the two-dimensional Euler equations. They used an approximate eigensystem analysis of a linearization around a computed solution of an airfoil flow.

In this paper we limit our investigation to approximations based on the true time-dependent system. Many methods use a modified form of the system, as for example in [14] where a scaling matrix multiplies the time-derivatives, or in calculations using the common practice where different time-steps are used in different parts of the domain.

An interesting result is that in many cases upwind differencing gives much faster convergence than centered differencing. This effect is not only due to the numerical dissipation. In fact, centered differencing with arbitrary large artificial dissipation added, does not give as good a convergence rate as upwind differencing.

This is important, since upwind differencing is used by many methods, in particular those designed for shock computations; see, for example, the review by Turkel [13] and the references therein, e.g. [1], [5], [11], [12]. All these methods reduce to one and the same method for the model problems treated in this paper.

In Section 2 we discuss how the differential operator and the boundary operators affect the convergence rate. The energy method and the Laplace transform are introduced.

Section 3 contains a study of the scalar advection equation and the corresponding approximations. A family of difference schemes is shown to have solutions which converge at an exponential rate. The standard upwind differencing produces the fastest convergence.

In Sections 4 and 5 the isentropic Euler equations are treated. Theoretical convergence estimates are given, and numerical experiments are presented. We also give a simple example showing that prescribing the characterstic quantities at the boundaries may actually produce a solution with faster convergence to steady state than prescribing boundary conditions which are equivalent to the pure initial value problem. Thus, in this case the simpler characteristic boundary conditions are more effective than higher-order radiation boundary conditions.

2. The Differential Equations. In this paper we will treat two different model problems describing scalar advection and homentropic flow in two space dimensions. However, we shall first discuss convergence of the solution to a steady state in more general terms.

Consider the following linear system of partial differential equations,

$$
\frac{\partial u}{\partial t}+\sum_{\nu=1}^{d} A_{\nu} \frac{\partial u}{\partial x_{\nu}}+A_{0} u=F, \quad x=\left(x_{1}, \ldots, x_{d}\right)^{T} \in \Omega, 0 \leqslant t
$$

Here $u$ and $F$ are $n$-component vectors, $A_{\nu}, \nu=0,1, \ldots, d$, are $n \times n$ matrices. This system requires initial- and boundary conditions

$$
\begin{gathered}
u(x, 0)=f(x), \quad x \in \Omega, \\
B(x, t) u(x, t)=g(x, t), \quad x \in \partial \Omega,
\end{gathered}
$$

where $B$ is a rectangular matrix. 
We are interested in problems (2.1) where the solutions converge uniquely to a steady state when $t \rightarrow \infty$. A necessary condition for convergence is that for problem (2.1) with $F=0, g=0$ the solution for any initial values converges to zero as $t \rightarrow \infty$.

There are essentially two phenomena causing the energy decay required for reaching a steady state.

(A) Internal decay mechanism created by variable coefficients $A_{\nu}=A_{\nu}(x, t)$ and the presence of lower-order terms $A_{0}$. This is to be distinguished from the dissipation mechanism in parabolic problems, where the decay rate depends on the frequency content of the solution. The decay rate for our type of hyperbolic problem is exponential.

Let $\Omega$ be the unit cube, and assume periodic boundary conditions and symmetric matrices $A_{\nu}, \nu=1, \ldots, d$. The scalar product and the norm are defined by

$$
(u, v)=\int_{\Omega} u^{*} v d x, \quad\|u\|^{2}=(u, u) .
$$

Integration by parts gives

$$
\frac{d}{d t}\|u\|^{2}=-2 \operatorname{Re}(u, D u), \quad D=A_{0}-\frac{1}{2} \sum_{\nu=1}^{d} \frac{\partial A_{\nu}}{\partial x_{\nu}},
$$

which implies that the decay rate is determined by $D$. We get

$$
e^{-d^{*} t}\|u(\cdot, 0)\| \leqslant\|u(\cdot, t)\| \leqslant e^{-d_{*} t}\|u(\cdot, 0)\|,
$$

where the constants $d_{*}, d^{*}$ satisfy

$$
2 d_{*} I \leqslant D+D^{*} \leqslant 2 d^{*} I .
$$

(Of course, we need $d_{*}>0$.)

(B) Boundary decay mechanism, which is determined by the boundary matrix $B$ together with the matrices $A_{\nu}$. When the integration by parts technique used above is applied to the nonperiodic case, boundary terms will remain. Let

$$
\hat{A}=\sum_{\nu=1}^{d} n_{\nu} A_{\nu}
$$

for $x \in \delta \Omega$, where $n=\left(n_{1}, \ldots, n_{d}\right)$ is the exterior normal to the boundary $\delta \Omega$ of $\Omega$. Then the extra boundary terms are given by the boundary integral $\int_{\delta \Omega} u^{*} \hat{A} u d S$, and there are two possibilities:

(1) $u^{*} \hat{A} u$ is positive for some vectors $u$ satisfying the boundary conditions. In this case the energy method fails to show even well-posedness of the problem.

(2) $u^{*} \hat{A} u$ is nonpositive for all vectors $u$ satisfying the boundary conditions. This is sufficient for well-posedness of the problem. However, not even the stronger condition

$$
u^{*} \hat{A} u \leqslant-\alpha u^{*} u, \quad \alpha>0,
$$

is sufficient for proving convergence to steady state as $t \rightarrow \infty$, since the boundary terms may vanish even if the solution in the interior has not reached a steady state. Such an example is given below. 
Example 2.1.

$$
\frac{\partial u}{\partial t}-y \frac{\partial u}{\partial x}+x \frac{\partial u}{\partial y}=0, \quad-1 \leqslant x \leqslant 1,-1 \leqslant y \leqslant 1, t>0
$$

Boundary conditions:

$$
\begin{array}{rlrl}
u=0 \text { for }: & x=1, & & 0<y<1, \\
& y=1, & & -1<x<0, \\
x & =-1, & -1<y<0, \\
y & =-1, & 0<x<1 .
\end{array}
$$

The solution is given by a rotation around the origin, and thus there is no convergence to steady state in general.

We saw above that it is important for convergence to steady state that energy in the solution is transported to the boundary, such that the boundary conditions can cause the norm $\|u(\cdot, t)\|$ to decay. This problem has been studied extensively in the following form: Is there a decay law

$$
\|u(\cdot, t)\|_{\Omega^{\prime}} \leqslant f(t),
$$

where $f(t) \rightarrow 0$ as $t \rightarrow \infty$ and $\Omega^{\prime}$ is a subset of $\Omega$ ? Very often, $\Omega$ is an exterior domain and $\Omega^{\prime}$ is bounded. Different conditions on the domain and the differential operator are derived in order to guarantee that the energy is not trapped in $\Omega^{\prime}$ and that there exists a decay law. See, e.g., [7], [8], [9].

We shall use the Laplace transform technique for obtaining estimates of the decay rate of the solution to the homogeneous problem. This technique is also used by Kreiss to prove well-posedness [6]. Let $\hat{u}(x, s)=\mathscr{L} u(x, t)$ be the Laplace transform of $u$, where $\mathscr{L}$ is defined by

$$
\hat{u}(x, s)=\int_{0}^{\infty} e^{-s t} u(x, t) d t .
$$

The inverse Laplace operator $\mathscr{L}^{-1}$ is formally given by

$$
u(x, t)=\mathscr{L}^{-1} \hat{u}(x, s)=\frac{1}{2 \pi i} \int_{\alpha-i \infty}^{\alpha+i \infty} e^{t s} \hat{u}(x, s) d s .
$$

We also need Parseval's relation

$$
\int_{0}^{\infty} e^{2 \alpha t}|u(x, t)|^{2} d t=2 \pi \int_{-\infty}^{\infty}|\hat{u}(x,-\alpha+i \beta)|^{2} d \beta,
$$

where the integrals are assumed to exist. We shall consider $x$-variable coefficients $A_{\nu}=A_{\nu}(x), B=B(x)$ for the homogeneous problem with $F=0, g=0$. The Laplace-transformed problem (2.1), for which we assume an existing classical solution with uniformly bounded derivatives, is

$$
\begin{gathered}
s \hat{u}+P \hat{u}=f, \quad x \in \Omega, \\
B \hat{u}=0, \quad x \in \partial \Omega,
\end{gathered}
$$

where

$$
P=\sum_{\nu=1}^{d} A_{\nu} \frac{\partial}{\partial x_{\nu}}+A_{0}
$$


The method for estimating the decay rate is based on the spectral properties of $P$. Assume that the problem (2.1) is well posed such that

$$
\int_{0}^{\infty} e^{-2 \alpha_{0} t}\|u(\cdot, t)\|^{2} d t<\infty \text { for some constant } \alpha_{0} .
$$

Then by Parseval's relation (2.2) the integral

$$
\int_{-\infty}^{\infty}\left|\hat{u}\left(x, \alpha_{0}+i \beta\right)\right|^{2} d \beta
$$

is also finite. We have the following result:

If the spectrum of the differential operator $P$ is disjoint from the strip $-\alpha_{1} \leqslant \operatorname{Re} s$ $\leqslant \alpha_{0}, \alpha_{1}>0$, such that for the solution to (2.3), $\mathscr{L}^{-1} \hat{u}$ is well defined in that strip, then $u$ decreases exponentially, i.e., for any constant $\alpha, \alpha>\alpha_{1}$,

$$
\|u(\cdot, t)\| \leqslant c e^{-\alpha t}\|f(\cdot)\| .
$$

The proposition follows from the fact that

$$
\int_{-\infty}^{\infty}\left|\hat{u}\left(x,-\alpha_{1}+i \beta\right)\right|^{2} d \beta
$$

is finite, and by applying Parseval's relation (2.2) with $\alpha=\alpha_{1}$. From the assumption of bounded time-derivatives, (2.5) follows immediately.

We have so far considered linear problems only. The analysis is in many cases relevant also for the computation of solutions to nonlinear problems in the important case where the solution is close to steady state and linearization is justifiable. If the solution contains shocks, there is, however, an extra source of energy decay that should be analyzed separately. We shall not deal with the special features of shocks in this paper.

3. The Advection Equation. We shall first use the advection equation as the simplest possible model equation which describes phenomena like pure transport of supersonic flow in more general hyperbolic problems. The corresponding differential equations have the property that their solutions in the typical case of a finite domain converge to a steady state after a finite time.

Model Problem I. The advection equation

$$
\begin{aligned}
& \text { (a) } \partial u / \partial t+\partial u / \partial x=0, \quad 0 \leqslant x \leqslant 1,0 \leqslant t \\
& \text { (b) } u(0, t)=0 \\
& \text { (c) } u(x, 0)=f(x)
\end{aligned}
$$

The solution to this problem is given by

$$
u(x, t)= \begin{cases}f(x-t), & x \geqslant t \\ 0, & x<t .\end{cases}
$$

The steady state $u \equiv 0$ is reached at $t=1$. When analyzing the corresponding difference approximation we shall use the Laplace transformation technique outlined in Section 2. Let us apply the same technique to the continuous problem (3.1).

The transformed problem is

$$
\begin{aligned}
& \text { (a) } s \hat{u}+\partial \hat{u} / \partial x=f, \quad 0 \leqslant x \leqslant 1, \\
& \text { (b) } \hat{u}(0, s)=0,
\end{aligned}
$$


which has the solution

$$
\hat{u}(x, s)=\int_{0}^{x} e^{-s(x-\xi)} f(\xi) d \xi
$$

Since (3.1) is well posed, we have

$$
I=\int_{-\infty}^{\infty}\left|\hat{u}\left(x, \alpha_{0}+i \beta\right)\right|^{2} d \beta=\int_{-\infty}^{\infty}\left|\int_{0}^{x} e^{-\left(\alpha_{0}+i \beta\right)(x-\xi)} f(\xi) d \xi\right|^{2} d \beta<\infty
$$

But

$$
I=\int_{-\infty}^{\infty}\left|e^{-\left(\alpha_{0}+\alpha_{1}\right) x} \int_{0}^{x} e^{-\left(-\alpha_{1}+i \beta\right)(x-\xi)} \tilde{f}(\xi) d \xi\right|^{2} d \beta
$$

where $\tilde{f}(\xi)=e^{\left(\alpha_{0}+\alpha_{1}\right) \xi} f(\xi)$.

Since the problem is well posed for all initial functions $\tilde{f}(x), I$ is finite, and we can get any decay rate according to the result in Section 2. We have actually shown that the differential operator $\partial / \partial x$ does not have any spectrum.

Consider next the semidiscrete approximation of (3.1),

$$
\begin{array}{ll}
\text { (a) } \partial u_{j} / \partial t+Q u_{j}=0, & j=1,2, \ldots, N-1,0 \leqslant t, \\
\text { (b) } u_{0}(t)=0, & \\
\text { (c) } B_{r} u_{N}(t)=0, & \\
\text { (d) } & u_{j}(0)=f_{j},
\end{array}
$$

where $u_{j}(t)$ approximates $u\left(x_{j}, t\right), x_{j}=j \Delta x$. The operator $Q$ is the general consistent 3-point difference operator parametrized in the form

$$
Q=D_{0}-\frac{c}{2} \Delta x D_{+} D_{-.}
$$

The standard notation for difference operators is used, i.e.,

$$
D_{0} u_{j}=\frac{1}{2 \Delta x}\left(u_{j+1}-u_{j-1}\right), \quad D_{ \pm} u_{j}= \pm \frac{1}{\Delta x}\left(u_{j \pm 1}-u_{j}\right) .
$$

$B_{r}$ is the $r$ th order extrapolation operator, i.e.,

$$
B_{r} u_{j}=\left(I-E^{-1}\right)^{r} u_{j}, \quad E^{-1} u_{j}=u_{j-1} .
$$

Our purpose is now to investigate the rate of convergence to steady state.

The spectral properties of the operator $Q$ coupled to the boundary conditions (3.4b) and (3.4c) implies the following result.

THEOREM 3.1. For any $c \geqslant 0$ the solution of (3.4) decays exponentially in time if $\Delta x$ is small enough.

Proof. We Laplace-transform the differential-difference equation (3.4) and consider the homogeneous problem

$$
\begin{aligned}
& \text { (a) } s \hat{u}_{j}+Q \hat{u}_{j}=0, \quad j=1,2, \ldots, N-1, \\
& \text { (b) } \hat{u}_{0}=0 \\
& \text { (c) } B_{r} \hat{u}_{N}=0
\end{aligned}
$$


where the first equation is

$$
s \hat{u}_{j}+\frac{1}{2 \Delta x}\left(\hat{u}_{j+1}-\hat{u}_{j-1}\right)-\frac{c}{2 \Delta x}\left(\hat{u}_{j+1}-2 \hat{u}_{j}+\hat{u}_{j-1}\right)=0 .
$$

We want to show that there is no nontrivial solution for $\operatorname{Re} s \geqslant 0$. Consider first the case $c=1$. The equation (3.5a) is then

$$
s \hat{u}_{j}+\frac{1}{\Delta x}\left(\hat{u}_{j}-\hat{u}_{j-1}\right)=0,
$$

with the general solution $\hat{u}_{j}=\alpha \kappa^{j}$, where $\kappa$ is given by

$$
s \kappa+\frac{1}{\Delta x}(\kappa-1)=0 \text {. }
$$

The boundary condition (3.5b) shows that there is only the trivial solution $\hat{u}_{j}=0$. For $c \neq 1$ the solution of $(3.5 \mathrm{a})$ is of the form

$$
\hat{u}_{j}=\alpha \kappa_{1}^{j}+\beta \kappa_{2}^{j},
$$

where $\kappa_{1} \neq \kappa_{2}$ are the solutions of the equation

$$
s \kappa+\frac{1}{2 \Delta x}\left(\kappa^{2}-1\right)-\frac{c}{2 \Delta x}(\kappa-1)^{2}=0 .
$$

For simplicity we rescale $s$ such that $s \rightarrow s / 2 \Delta x$. (3.6) can then be written

$$
\kappa^{2}+\frac{s+2 c}{1-c} \kappa-\frac{1+c}{1-c}=0
$$

which has the solutions

$$
\kappa_{1,2}=-\frac{s+2 c}{2(1-c)} \pm \sqrt{\left(\frac{s+2 c}{2(1-c)}\right)^{2}+\frac{1+c}{1-c}} .
$$

The $\kappa$-roots of (3.7) coincide if

$$
(s+2 c)^{2}+4\left(1-c^{2}\right)=0,
$$

which is only possible for $s= \pm 2 i$ and $c=0$ when $\operatorname{Re} s \geqslant 0$ and $c \geqslant 0$. This case is analyzed later.

Consider $\operatorname{Re} s \geqslant 0, c>0$ and $c \neq 1$. The semidiscrete approximation (3.4) is dissipative, which implies

$$
\operatorname{Re} s<0 \text { for } \kappa=e^{i \omega}, \omega \text { real, } \omega \neq 0, \pm 2 \pi, \pm 4 \pi, \ldots,
$$

where $s$ is given by (3.7). Hence, there is no solution $\kappa$ of (3.7) with $|\kappa|=1$ when $\operatorname{Re} s>0$.

For $s=M>0, M \rightarrow+\infty$, we get $\kappa_{1} \rightarrow 0, \kappa_{2} \rightarrow+\infty$ and thus, due to continuity of the roots, $\left|\kappa_{1}\right|<1$ for all $\operatorname{Re} s>0$. Since $\kappa_{1} \kappa_{2}=-(1+c) /(1-c)$ we get

$$
\left|\kappa_{2}\right| \geqslant \frac{1+c}{1-c} \text {. }
$$

The general solution is inserted into the boundary conditions, and a linear system of equations is obtained for $\alpha, \beta$. A nontrivial solution exists only if the determinant vanishes, i.e.,

$$
\operatorname{det}\left|\begin{array}{cc}
1 & 1 \\
\kappa_{1}^{N-r}\left(\kappa_{1}-1\right)^{r} & \kappa_{2}^{N-r}\left(\kappa_{2}-1\right)^{r}
\end{array}\right|=0
$$


or, equivalently,

$$
\left(\frac{\kappa_{2}}{\kappa_{1}}\right)^{N-r}=\left(\frac{\kappa_{1}-1}{\kappa_{2}-1}\right)^{r} .
$$

No nontrivial solution is possible for any fixed $r$ and $c$ if $N$ is large enough $(\Delta x$ small enough) and

$$
\begin{gathered}
\left|\frac{\kappa_{1}-1}{\kappa_{2}-1}\right|^{r} \text { bounded, since } \\
\left|\frac{\kappa_{2}}{\kappa_{1}}\right|^{N-r} \geqslant\left|\frac{1+c}{1-c}\right|^{N-r} \rightarrow+\infty, \quad N \rightarrow+\infty .
\end{gathered}
$$

When $c>0$, the above relations are valid also for $\operatorname{Re} s=0$.

Consider finally $c=0$ and $\operatorname{Re} s \geqslant 0$. The continuity of the spectrum implies that only $\operatorname{Re} s=0$ needs to be studied. The equation (3.7) becomes

$$
\kappa^{2}+s \kappa-1=0, \quad s=2 i \omega, \omega \text { real, }
$$

and the roots are $\kappa_{1,2}=-i \omega \pm \sqrt{1-\omega^{2}}$.

For $\omega \neq \pm 1$ the determinant condition (3.8) is necessary for the existence of a nontrivial solution.

For $|\omega|<1$ both roots $\kappa_{1}, \kappa_{2}$ are on the unit circle.

Equation (3.8) gives

$$
\left|\frac{-i \omega+\sqrt{1-\omega^{2}}-1}{-i \omega-\sqrt{1-\omega^{2}}-1}\right|=1
$$

which is not possible.

For $\omega= \pm 1$ the equation (3.9) has double roots $\kappa=-i$ and $\kappa=i$ corresponding to the eigenvalues $s=-2 i$ and $s=2 i$. The general solution of the homogeneous problem $(3.5 \mathrm{a})$ is

$$
\hat{u}_{j}=(\alpha+\beta j)( \pm i)^{j} .
$$

The boundary condition $\hat{u}_{0}=0$ implies $\alpha=0$, and we also have $\beta=0$ since

$$
\left(I-E^{-1}\right)^{r} j( \pm i)^{j} \neq 0 .
$$

In the final case $\omega>1$, the roots $\kappa_{1}$ and $\kappa_{2}$ are both purely imaginary,

$$
\kappa_{1,2}=i\left(-\omega \pm \sqrt{\omega^{2}-1}\right) \text {. }
$$

The relations $\left|\kappa_{1}\right|<\left|\kappa_{2}\right|,\left|\kappa_{1}-1\right|<\left|\kappa_{2}-1\right|$ contradict (3.8) and there is no nontrivial solution.

This proves that the system (3.4) only has exponentially decaying solutions.

The whole spectrum of $Q$ was computed using a standard eigenvalue routine for the case $c=0$, i.e., $Q=D_{0}$. The linear extrapolation procedure was used as boundary condition at $x=1$. Figure 3.1 shows clearly how one end of the spectrum (for $-Q$ ) approaches the imaginary axis as $\Delta x$ gets smaller. Actually, one can show that the distance between the imaginary axis and the right end of the spectrum is proportional to $(\Delta x)^{2}$, indicating a very poor convergence rate for steady state calculations using centered nondissipative difference operators. As a constrast, the upwind difference operator $-D_{-}$obtained for $c=1$ has the only eigenvalue $-1 / \Delta x$ (but with multiplicity $N$ ). 


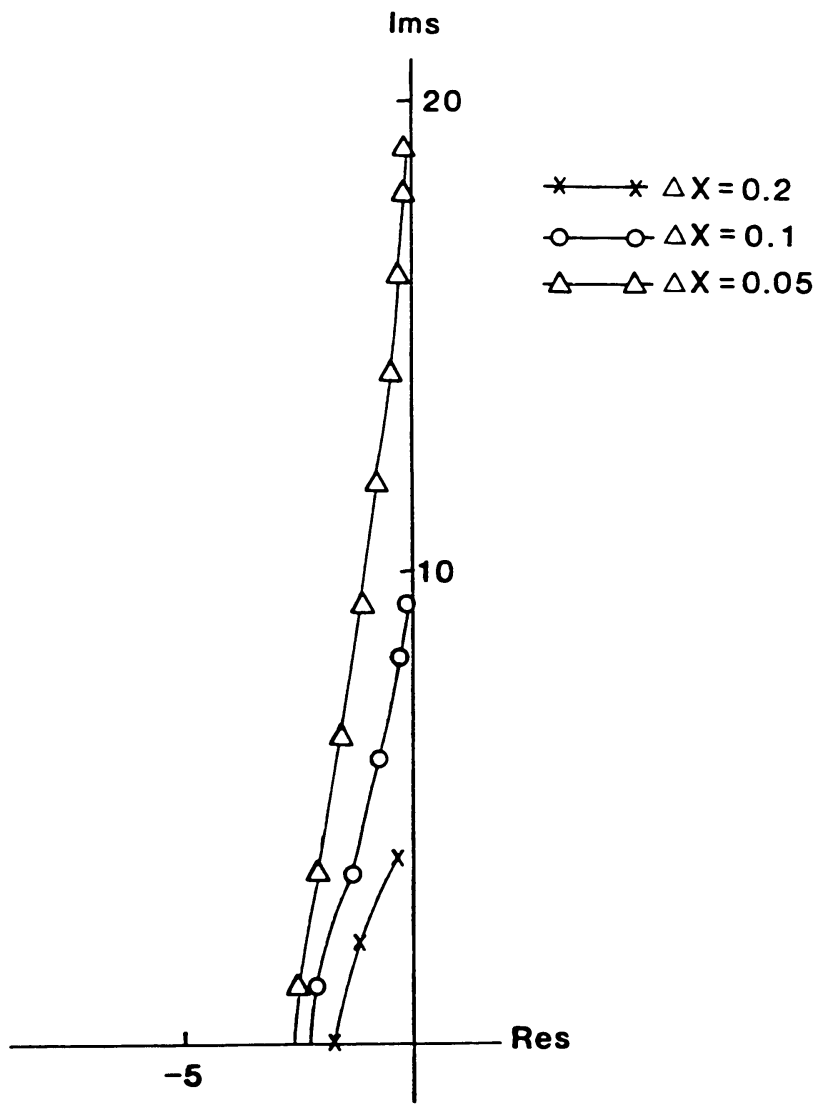

FIGURE 3.1

Spectrum of $-D_{0}$ with linear extrapolation at the boundary.

(Upper half of the spectrum displayed.)

As we have seen, the introduction of boundary conditions has a dissipative effect on the nondissipative operator $-D_{0}$. For comparison we shall show what effect the introduction of the dissipative term $c \Delta x D_{+} D_{-} / 2$ has in the periodic case. The eigenvalues for $-Q$ are, in the periodic case,

$$
s=\frac{1}{\Delta x}\left(-i \sin 2 \pi \omega \Delta x-2 c \sin ^{2} \pi \omega \Delta x\right), \quad \omega=0,1, \ldots, N-1 .
$$

Figure 3.2 shows the periodic case for $c=0$ and $c=0.2$, and the boundary condition case for $-D_{0}$ (shown also in Figure 3.1). In all cases the step-length is $\Delta x=0.1$.

The general convergence rate is governed by the eigenvalue $\hat{s}$ with the largest real part. In Figure 3.3, $\operatorname{Re} \hat{s}$ is presented as a function of $c$, and a very sharp peak is obtained at $c=1$ corresponding to the upwind operator $D_{-}$.

The use of second-order accurate upwind differencing requires an extra numerical inflow boundary condition for nonperiodic problems. If that condition is given by first-order upwind differencing, $\hat{s}$ is again equal to $-1 / \Delta x$. However, contrary to the previous case, this eigenvalue is simple. 


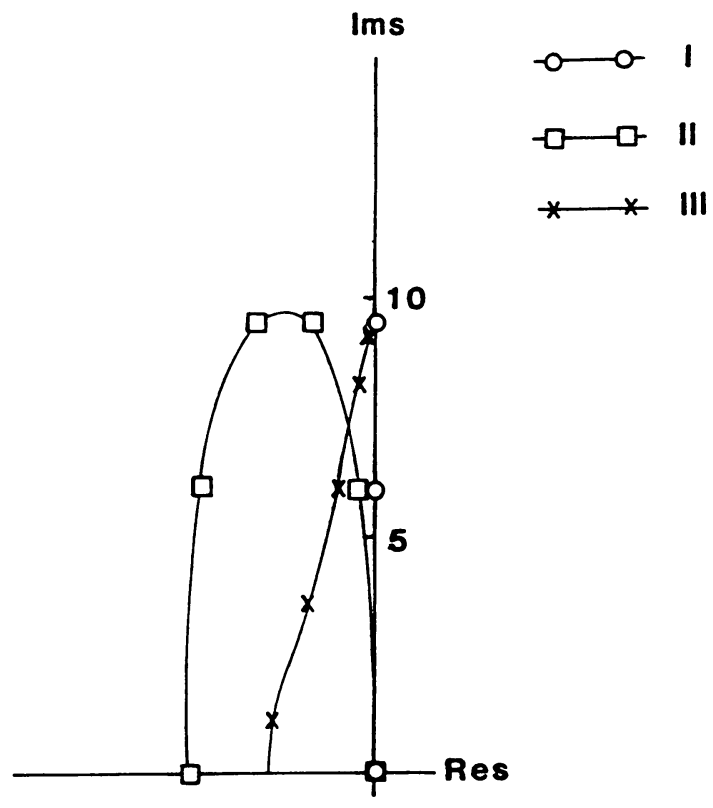

Figure 3.2

Spectrum of $-D_{0}+c \Delta x D_{+} D_{-} / 2$ with periodic boundary conditions. I: $c=0$, II: $c=0.2$, and III: spectrum of $-D_{0}$ with linear extrapolation at the boundary. $\Delta x=0.1$. (Upper half of the spectrum displayed.)

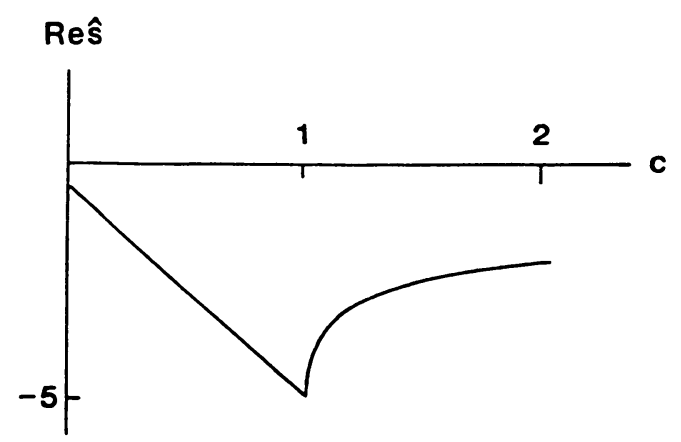

FIGURE 3.3

Largest real part $(\operatorname{Re} \hat{s})$ of the eigenvalues to $-D_{0}+$ $c \Delta x D_{+} D_{-} / 2$ as a function of $c$. Linear extrapolation at the boundary for $c \neq 1\left(u_{0}=0, u_{N}=2 u_{N-1}-u_{N-2}\right) . \Delta x=0.2$.

4. The Euler Equations. In this section we shall consider inviscid isentropic flow in a channel. The Euler equations are

$$
\frac{\partial w}{\partial t}+A(w) \frac{\partial w}{\partial x}+B(w) \frac{\partial w}{\partial y}=0
$$

where

$$
w=\left(\begin{array}{l}
\rho \\
u \\
v
\end{array}\right), \quad A(w)=\left(\begin{array}{ccc}
u & \rho & 0 \\
c^{2} / \rho & u & 0 \\
0 & 0 & u
\end{array}\right), \quad B(w)=\left(\begin{array}{ccc}
v & 0 & \rho \\
0 & v & 0 \\
c^{2} / \rho & 0 & v
\end{array}\right)
$$

$u$ and $v$ are the velocities in the $x$-and $y$-directions, respectively. 


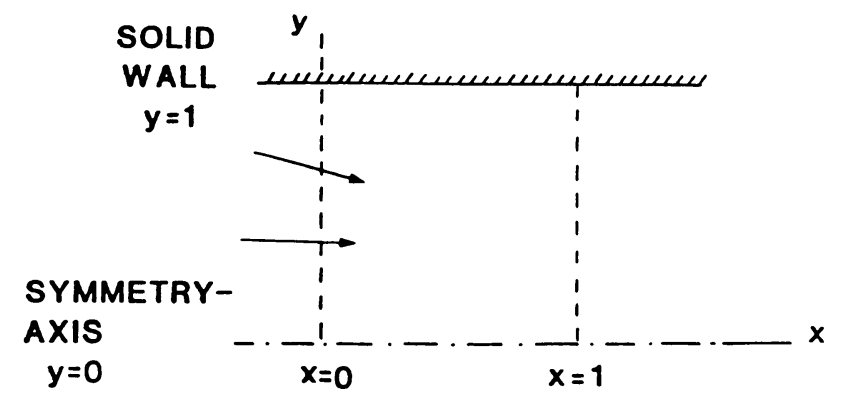

Figure 4.1

Computational domain for the Euler equations.

It has been assumed that the pressure $p$ and the density $\rho$ are related by an algebraic relation $p=p(\rho)$. The local speed of sound $c$ is defined by

$$
c^{2}=d p / d \rho .
$$

The equations (4.1) are defined on a domain corresponding to a channel with infinite extension in the $x$-direction according to Figure 4.1.

The system (4.1) is linearized around some constant state $\bar{w}$ and the variables are made dimensionless. The normalizing velocity is the $x$-component $u$, so that the diagonal of $A$ becomes the unit matrix. Since the $y$-component $v$ is zero at both horizontal boundaries, it is assumed that $\bar{v}=0$ in the constant state which we are linearizing around. In order to get symmetric coefficient matrices $A, B$, the velocity components are scaled by the factor $\bar{\rho} / \bar{c}$. For convenience, the original notation $w=(\rho, u, v)^{T}$ is kept also for the new variables, and we get the linearized Euler equations with constant coefficients

$$
\frac{\partial w}{\partial t}+A \frac{\partial w}{\partial x}+B \frac{\partial w}{\partial y}=0, \quad 0 \leqslant x, y \leqslant 1,0 \leqslant t
$$

where

$$
w=\left(\begin{array}{l}
\rho \\
u \\
v
\end{array}\right), \quad A=\left(\begin{array}{lll}
1 & c & 0 \\
c & 1 & 0 \\
0 & 0 & 1
\end{array}\right), \quad B=\left(\begin{array}{lll}
0 & 0 & c \\
0 & 0 & 0 \\
c & 0 & 0
\end{array}\right) .
$$

The supersonic case is defined by the condition $c<1$. In that case the boundary conditions are

$$
\left\{\begin{array}{l}
\rho(0, y, t)=g^{\mathrm{I}}(y, t), \\
u(0, y, t)=g^{\mathrm{II}}(y, t), \\
v(0, y, t)=g^{\mathrm{III}}(y, t), \\
v(x, 1, t)=0
\end{array}\right.
$$

At the symmetry axis $y=0$ the conditions are

$$
\left\{\begin{array}{l}
\partial \rho(x, 0, t) / \partial y=0 \\
\partial u(x, 0, t) / \partial y=0 \\
v(x, 0, t)=0
\end{array}\right.
$$

As in the scalar advection case, the solutions decay. 
THEOREM 4.1. The solutions to the supersonic problem (4.2) with homogeneous boundary conditions (4.3), (4.4) vanish for $t>1 /(1-c)$.

Proof. Introduce the new vector $\tilde{w}$ by

$$
\tilde{w}=e^{\alpha(t-\beta x)} w, \quad \alpha>0, \beta>0 .
$$

The equation (4.2) then becomes

$$
\frac{\partial \tilde{w}}{\partial t}+A \frac{\partial \tilde{w}}{\partial x}+B \frac{\partial \tilde{w}}{\partial y}+\alpha(\beta A-I) \tilde{w}=0,
$$

and we get for $c<1$,

$$
\begin{aligned}
\frac{1}{2} & \frac{d}{d t}\|\tilde{w}\|^{2}=\left(\tilde{w}, \frac{\partial \tilde{w}}{\partial t}\right)=-\left(\tilde{w}, A \frac{\partial \tilde{w}}{\partial x}\right)-\left(\tilde{w}, B \frac{\partial \tilde{w}}{\partial y}\right)-\alpha(\tilde{w},(\beta A-I) \tilde{w}) \\
= & -\frac{1}{2} \int_{0}^{1}\left(\tilde{\rho}(1, y, t)^{2}+\tilde{u}(1, y, t)^{2}+2 c \tilde{\rho}(1, y, t) \tilde{u}(1, y, t)+\tilde{v}(1, y, t)^{2}\right) d y \\
& -\alpha(\tilde{w},(\beta A-I) \tilde{w}) \leqslant 0 \quad \text { if } \beta A \geqslant I .
\end{aligned}
$$

( $A$ is positive definite. The norm is defined by $\|w\|^{2}=(w, w)=\int_{0}^{1} \int_{0}^{1} w^{T} w d x d y$.)

Hence,

$$
\begin{aligned}
\|w(\cdot, \cdot, t)\| & =e^{-\alpha t}\left[\int_{0}^{1} \int_{0}^{1} e^{2 \alpha \beta x} \tilde{w}(x, y, t)^{2} d x d y\right]^{1 / 2} \\
& \leqslant e^{-\alpha t+\alpha \beta}\|\tilde{w}(\cdot, \cdot, 0)\| \leqslant e^{-\alpha(t-\beta)}\|w(\cdot, \cdot, 0)\| .
\end{aligned}
$$

The constant $\beta$ can be chosen equal to $1 /(1-c)$, and since $\alpha$ is arbitrary, the solution must vanish for $t>1 /(1-c)$.

We next turn to the subsonic case $c>1$. Two boundary conditions must be given at $x=0$ and one condition at $x=1$. We first consider specification of the Riemann invariants, which in the linear case corresponds to a specification of the characteristic variables:

$$
\left\{\begin{array}{l}
\rho(0, y, t)+u(0, y, t)=g^{\mathrm{I}}(y, t) \\
v(0, y, t)=g^{\mathrm{II}}(y, t) \\
\rho(1, y, t)-u(1, y, t)=g^{\mathrm{III}}(y, t) \\
v(x, 1, t)=0
\end{array}\right.
$$

It is easily shown by the energy method that these conditions give rise to a well-posed problem. In order to estimate the convergence rate to steady state, we want to compute the spectrum of the differential operator in space. To make that computation easier, we first apply the Fourier transform used in [2] for the same problem, i.e., we use the expansions

$$
\begin{aligned}
& \rho(x, y, t)=\sum_{\omega=0}^{\infty} \hat{\rho}_{\omega}(x, t) \cos \pi \omega y, \\
& u(x, y, t)=\sum_{\omega=0}^{\infty} \hat{u}_{\omega}(x, t) \cos \pi \omega y, \\
& v(x, y, t)=\sum_{\omega=1}^{\infty} \hat{v}_{\omega}(x, t) \sin \pi \omega y .
\end{aligned}
$$


The introduction of these expansions into (4.1) gives for each $\omega$

$$
\frac{\partial \hat{w}}{\partial t}+A \frac{\partial \hat{w}}{\partial x}+\pi \omega \hat{B} \hat{w}=0
$$

where

$$
\hat{w}=\left(\begin{array}{c}
\hat{\rho}_{\omega} \\
\hat{u}_{\omega} \\
\hat{v}_{\omega}
\end{array}\right), \quad \hat{B}=\left(\begin{array}{ccc}
0 & 0 & c \\
0 & 0 & 0 \\
-c & 0 & 0
\end{array}\right)
$$

The spectrum is given by the set of $s$-values which satisfy

$$
s \hat{w}+A \frac{\partial \hat{w}}{\partial x}+\pi \omega \hat{B} \hat{w}=0
$$

for nontrivial vectors $\hat{w}$ which fulfill the homogeneous boundary conditions. The general solution to (4.6) can be written in the form

$$
\hat{w}=\sigma_{1}\left(\begin{array}{c}
0 \\
\pi \omega c \\
c s
\end{array}\right) e^{\kappa_{1} x}+\sigma_{2}\left(\begin{array}{c}
s+\kappa_{2} \\
-c \kappa_{2} \\
\pi \omega c
\end{array}\right) e^{\kappa_{2} x}+\sigma_{3}\left(\begin{array}{c}
s+\kappa_{3} \\
-c \kappa_{3} \\
\pi \omega c
\end{array}\right) e^{\kappa_{3} x}
$$

Here the coefficients $\sigma_{1}, \sigma_{2}, \sigma_{3}$ are to be determined by the boundary conditions, the exponential coefficients $\kappa_{1}, \kappa_{2}, \kappa_{3}$ are the distinct roots of the characteristic equation

$$
\operatorname{det}\left(\begin{array}{ccc}
s+\kappa & c \kappa & \pi \omega c \\
c \kappa & s+\kappa & 0 \\
-\pi \omega c & 0 & s+\kappa
\end{array}\right)=0
$$

(For multiple roots $\kappa_{j}$, the general solution (4.7) has a different form if the coefficient vectors are linearly dependent.)

The roots of (4.8) are given by

$$
\left\{\begin{array}{l}
\kappa_{1}=-s \\
\kappa_{2,3}=\frac{1}{c^{2}-1}\left[s \pm \sqrt{\left(c^{2}-1\right)(\pi \omega c)^{2}+c^{2} s^{2}}\right] .
\end{array}\right.
$$

The general solution (4.7) is inserted into the boundary conditions

$$
\begin{cases}\hat{\rho}+\hat{u}=\hat{v}=0 & \text { for } x=0, \\ \hat{\rho}-\hat{u}=0 & \text { for } x=1,\end{cases}
$$

giving

$$
\left\{\begin{array}{l}
\pi \omega c \sigma_{1}+\left[s-\kappa_{2}(c-1)\right] \sigma_{2}+\left[s-\kappa_{3}(c-1)\right] \sigma_{3}=0 \\
s \sigma_{1}+\pi \omega \sigma_{2}+\pi \omega \sigma_{3}=0 \\
-\pi \omega c e^{\kappa_{1}} \sigma_{1}+\left[s+\kappa_{2}(c+1)\right] e^{\kappa_{2}} \sigma_{2}+\left[s+\kappa_{3}(c+1)\right] e^{\kappa_{3}} \sigma_{3}=0
\end{array}\right.
$$

which has a nontrivial solution $\sigma_{1}, \sigma_{2}, \sigma_{3}$ if and only if

$$
\begin{gathered}
s\left\{\left[s-\kappa_{3}(c-1)\right]\left[s+\kappa_{2}(c+1)\right] e^{\kappa_{2}}\right. \\
\left.-\left[s-\kappa_{2}(c-1)\right]\left[s+\kappa_{3}(c+1)\right] e^{\kappa_{3}}\right\} \\
+\pi^{2} \omega^{2} c\left\{\left[s+\kappa_{3}(c+1)\right] e^{\kappa_{3}}-\left[s+\kappa_{2}(c+1)\right] e^{\kappa_{2}}\right. \\
\left.\quad-e^{\kappa_{1}}\left(\kappa_{3}-\kappa_{2}\right)(c-1)\right\}=0 .
\end{gathered}
$$


There are four points in the $s$-plane where the general form (4.7) is not valid:

$$
\begin{array}{lll}
s=\pi \omega, & \kappa_{1}=\kappa_{2}=-\pi \omega, & \kappa_{3}=\pi \omega \frac{c^{2}+1}{c^{2}-1} ; \\
s=-\pi \omega, & \kappa_{1}=\kappa_{3}=\pi \omega, & \kappa_{2}=-\pi \omega \frac{c^{2}+1}{c^{2}-1} ; \\
s=\pi \omega i \sqrt{c^{2}-1}, & \kappa_{1}=-\pi \omega i \sqrt{c^{2}-1}, & \kappa_{2}=\kappa_{3}=\frac{\pi \omega i}{\sqrt{c^{2}-1}} ; \\
s=-\pi \omega i \sqrt{c^{2}-1}, & \kappa_{1}=\pi \omega i \sqrt{c^{2}-1}, & \kappa_{2}=\kappa_{3}=-\frac{\pi \omega i}{\sqrt{c^{2}-1}} .
\end{array}
$$

At these points the general solution consists of two exponentials, where one is multiplied by a polynomial in $x$ of degree one with vector coefficients. It can be shown that none of these exceptional points belongs to the spectrum.

(4.10) is a scalar equation in the complex variable $s$. It was solved by Newton's method using different initial values such that all solutions within a certain domain around the origin were obtained. The result is shown in Figure 4.2 for $c=2, \omega=1$, and $\omega=4$. The whole spectrum is for each $\omega$ contained in the left half-plane.

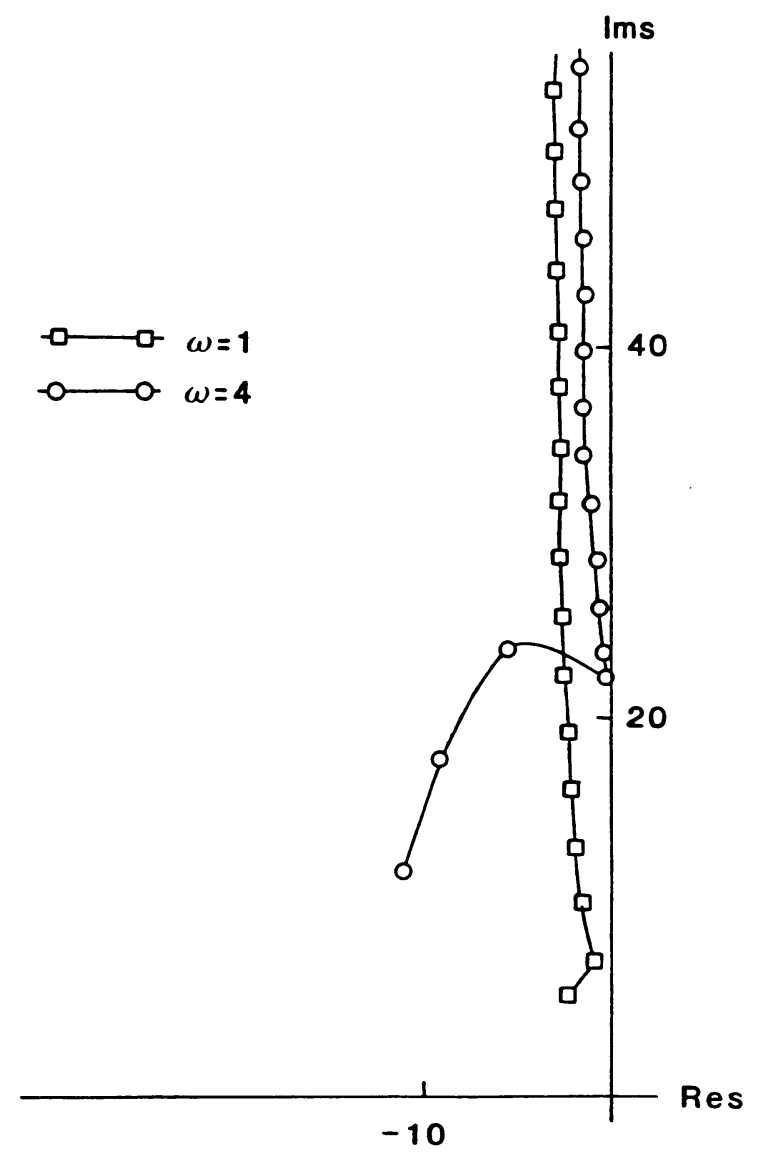

Figure 4.2

Spectrum for the Euler subsonic differential operator with characteristic boundary conditions. (Upper half of the spectrum displayed.) 
However, there is always one eigenvalue close to the imaginary axis, in fact, when $|\omega|$ increases, the distance goes to zero as $1 /|\omega|$.

We have also considered a different set of boundary conditions which may be more natural for those who have to provide the data. Instead of (4.5) we use

$$
\left\{\begin{array}{l}
u(0, y, t)=g^{\mathrm{I}}(y, t), \\
v(0, y, t)=g^{\mathrm{II}}(y, t), \\
\rho(1, y, t)=g^{\mathrm{II}}(y, t), \\
v(x, 1, t)=0
\end{array}\right.
$$

In [10] it was shown that these conditions give a well-posed problem. The spectrum was computed in the same way as above. The final equation corresponding to (4.10) is

$$
\left(\pi^{2} \omega^{2}+s \kappa_{2}\right)\left(s+\kappa_{3}\right) e^{\kappa_{3}}-\left(\pi^{2} \omega^{2}+s \kappa_{3}\right)\left(s+\kappa_{2}\right) e^{\kappa_{2}}=0,
$$

where $\kappa_{2}, \kappa_{3}$ are given by (4.9) as before. In this case the spectrum is purely imaginary. This reflects the fact that the $L_{2}$-energy of the system is conserved. Part of the spectrum is shown in Figure $5.3(\omega=1)$ where the spectrum of the discrete operator is also given.

In this section we have seen that the decay rate and thus the convergence rate as $t \rightarrow \infty$ may vary drastically depending on the flow field and the boundary conditions. For supersonic flow, the solutions vanish after finite time. For subsonic flow with boundary conditions (4.11), the energy does not decay at all, and with boundary conditions (4.5) the decay is slower than any exponential and depends on the smoothness of the solution. The higher frequencies (large $\omega$ ) decay at a slower rate than the lower frequencies (small $\omega$ ).

The case where the matrices $A$ and $B$ are linearized around zero velocities corresponds to the wave equation and has been studied extensively; see, e.g., [9]. The equations are

$$
\left\{\begin{array}{l}
\rho_{t}+c u_{x}+c w_{y}=0 \\
u_{t}+c \rho_{x}=0 \\
v_{t}+c \rho_{y}=0
\end{array}\right.
$$

From this system there follows the scalar wave equation

$$
\rho_{t t}=c^{2}\left(\rho_{x x}+\rho_{y y}\right) .
$$

In [7] and [8] it is proved, for example, that the energy in a bounded domain decays like $t^{-2}$ if the wave equation is solved in the exterior of a star-shaped domain for the Neumann and Dirichlet problems.

The boundary conditions (4.5) correspond to giving some of the characteristic quantities at the inflow and outflow boundaries. One may speculate in the possibility of choosing artificial boundary conditions that produce solutions which more accurately simulate flows in open channels. The hope would be for an improvement in the decay rate. We shall give a simple example showing that the exact open boundary condition, which is equivalent to the corresponding Cauchy problem, may actually be worse than giving the characteristic quantities. Consider the differential 
equations

$$
\left\{\begin{array}{l}
\frac{\partial u}{\partial t}+\frac{\partial u}{\partial x}+u=0, \\
\frac{\partial v}{\partial t}-\frac{\partial v}{\partial x}-u=0,
\end{array}\right.
$$

with initial conditions

$$
\begin{aligned}
& u(x, 0)=f(x), \\
& v(x, 0)=g(x), \quad 0 \leqslant x \leqslant 1,
\end{aligned}
$$

and characteristic boundary condition

$$
u(0, t)=0, \quad v(1, t)=0
$$

The solution

$$
\begin{array}{ll}
u(x, t)=e^{-t} f(x-t), & x-t>0 \\
u(x, t)=0, & x-t \leqslant 0 \\
v(x, t)=\int_{t-1+x}^{t} u(x+t-\tau, \tau) d \tau &
\end{array}
$$

vanishes after $t=2$.

With open boundary conditions to the right, the differential equation is valid in the domain $x>0, t>0$ with the initial values $f(x) \equiv g(x) \equiv 0$ for $x \geqslant 1$. The boundary condition is $u(0, t)=0$, and the solution is given by

$$
u(x, t)=e^{-t} f(x-t), \quad v(x, t)=\int_{0}^{t} u(x+t-\tau, \tau) d \tau .
$$

In the interval $0<x<1$ the solution decays exponentially with time, and hence slower than for the corresponding problem with characteristic boundary conditions.

5. Difference Approximations to the Euler Equations. In this section we shall study upwind difference methods for the Euler equations with constant coefficients as given in Section 4. The upwind differencing technique, in the scalar one-dimensional case, is based on the idea of using information only from that side which contains the data required for generating the solution in the continuous case. This technique is easily generalized to systems and to several space dimensions. We simply treat each term one-dimensionally, and separate the left- and the right-going characteristic variables.

Let $S$ and $T$ be the matrices that diagonalize $A$ and $B$ in (4.2), respectively, i.e.,

$$
S^{T} A S=\left(\begin{array}{ccc}
1+c & 0 & 0 \\
0 & 1-c & 0 \\
0 & 0 & 1
\end{array}\right), \quad T^{T} B T=\left(\begin{array}{ccc}
c & 0 & 0 \\
0 & 0 & 0 \\
0 & 0 & -c
\end{array}\right) .
$$

The one-dimensional problem

$$
\frac{\partial U}{\partial t}+A \frac{\partial U}{\partial x}=0
$$

is equivalent to

$$
\frac{\partial V}{\partial t}+S^{T} A S \frac{\partial V}{\partial x}=0, \quad V=S^{T} U
$$


which is a set of three scalar equations. With the projection operators $P_{+}, P_{-}$ defined corresponding to the sign of the eigenvalues, i.e., for $c>1$,

$$
P_{+}=\left(\begin{array}{ccc}
1 & 0 & 0 \\
0 & 0 & 0 \\
0 & 0 & 1
\end{array}\right), \quad P_{-}=\left(\begin{array}{ccc}
0 & 0 & 0 \\
0 & 1 & 0 \\
0 & 0 & 0
\end{array}\right),
$$

corresponding to the subsonic case, the first-order upwind scheme is

$$
P_{+} \frac{\partial V_{j}}{\partial t}+P_{+} S^{T} A S\left(D_{-} V_{j}\right)=0, \quad P_{-} \frac{\partial V_{j}}{\partial t}+P_{-} S^{T} A S\left(D_{+} V_{j}\right)=0 .
$$

To regain the original variables, we add the two equations together and multiply by $S$, which gives

$$
\frac{\partial U_{j}}{\partial t}+A_{+}\left(D_{-} U_{j}\right)+A_{-}\left(D_{+} U_{j}\right)=0
$$

where $A_{+}=S P_{+} S^{T} A, A_{-}=S P_{-} S^{T} A$.

The $y$-direction is treated analogously, and we obtain the upwind scheme for (4.2) of first order,

$$
\frac{\partial U_{j}}{\partial t}+Q U_{j}=0, \quad Q=A_{+} D_{-x}+A_{-} D_{+x}+B_{+} D_{-y}+B_{-} D_{+y},
$$

where the difference operators have been given proper subscripts to indicate the direction of differencing. Since in our case the flow is always subsonic in the $y$-direction, the coefficient matrices $B_{+}, B_{-}$are

$$
B_{+}=\frac{c}{2}\left(\begin{array}{ccc}
1 & 0 & 1 \\
0 & 0 & 0 \\
1 & 0 & 1
\end{array}\right), \quad B_{-}=\frac{c}{2}\left(\begin{array}{ccc}
-1 & 0 & 1 \\
0 & 0 & 0 \\
1 & 0 & -1
\end{array}\right)
$$

As in the continuous case, we shall use the special Fourier transform in order to simplify the spectral analysis. At any given time it is assumed that the solution has the form

$$
\begin{aligned}
\rho_{j k} & =\sum_{\omega=0}^{M} \hat{\rho}_{j}(\omega) \cos \pi \omega y_{k}, \\
u_{j k} & =\sum_{\omega=1}^{M} \hat{u}_{j}(\omega) \cos \pi \omega y_{k}, \\
v_{j k} & =\sum_{\omega=0}^{M} \hat{v}_{j}(\omega) \sin \pi \omega y_{k}, \quad k=0,1, \ldots, M, M=1 / \Delta y .
\end{aligned}
$$

An easy calculation shows that with $\eta=\pi \omega \Delta y$ we get the transformed system

where $\hat{Q}=A_{+} D_{-x}+A_{-} D_{+x}+\hat{B}$,

$$
\frac{\partial}{\partial t} \hat{U}_{j}(\omega)+\hat{Q} \hat{U}_{j}(\omega)=0
$$

$$
\hat{B}=\frac{c}{\Delta y}\left(\begin{array}{ccc}
1-\cos \eta & 0 & \sin \eta \\
0 & 0 & 0 \\
-\sin \eta & 0 & 1-\cos \eta
\end{array}\right) \text {. }
$$

The Supersonic Case $c<1$. If the original flow is supersonic in the $x$-direction, we have $c<1, A_{+}=A, A_{-}=0$, i.e., $\hat{Q}=A D_{-x}+\hat{B}$. The approximation is a differential in time-difference in space equation

$$
\frac{\partial}{\partial t} \hat{U}_{j}(\omega)+\left(\frac{1}{\Delta x} A+\hat{B}\right) \hat{U}_{j}(\omega)-\frac{1}{\Delta x} A \hat{U}_{j-1}(\omega)=0
$$


All boundary conditions are given at $x=0$, and in order to find the spectrum, we set $\hat{U}_{0}(\omega)=0$. Obviously, the eigenvalues of $\hat{Q}$ are given by

$$
\operatorname{det}\left(\frac{1}{\Delta x} A+\hat{B} s I\right)=0 \text {. }
$$

The three eigenvalues given by this equation were computed numerically for $\Delta x=\Delta y, c=1 / 2,0 \leqslant \eta<2 \pi$. The maximal real part of $s$ is shown in Figure 5.1, and it is seen that the matrix $\hat{B}$ actually forces the spectrum even further to the left compared to the one-dimensional case.

Since the use of upwind difference operators introduces dissipation, one may think that a change to centered differences in the $y$-direction should give a spectrum located further to the right. But this is true only for certain values of $\eta$ as shown in Figure 5.1.

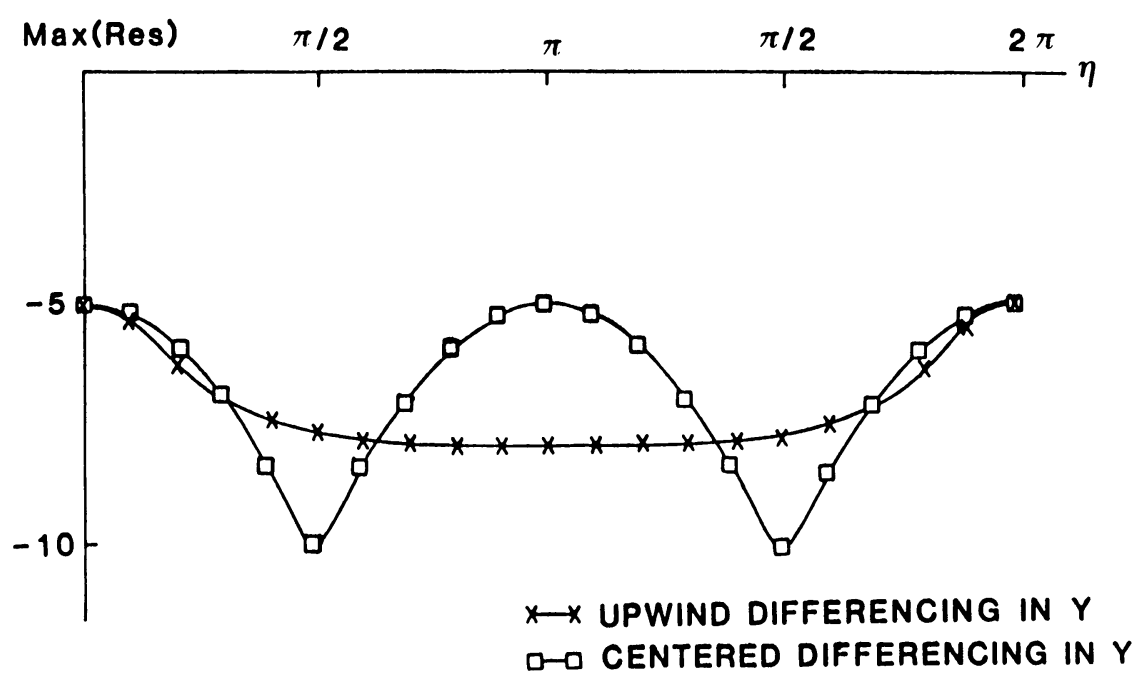

FIGURE 5.1

Largest real part $(\operatorname{Re} \hat{s})$ of the eigenvalues $s$ of the upwind Fourier-transformed difference approximation to the supersonic Euler differential operator is shown as a function of $\eta=\pi \omega \Delta y$, where $\omega$ is the dual variable to $y . \Delta x=\Delta y=0.1$. The speed of sound $c=0.5$.

The Subsonic Case $c>1$. For subsonic flow in the $x$-direction, we have $c>1$, and the coefficient matrices $A_{+}, A_{-}$are

$$
A_{+}=\frac{1}{2}\left(\begin{array}{ccc}
1+c & 1+c & 0 \\
1+c & 1+c & 0 \\
0 & 0 & 2
\end{array}\right), \quad A_{-}=\frac{1}{2}\left(\begin{array}{ccc}
1-c & c-1 & 0 \\
c-1 & 1-c & 0 \\
0 & 0 & 0
\end{array}\right)
$$

Two boundary conditions must be given at $x=0$, and one condition at $x=1$. We shall consider the two different sets of boundary conditions given in Section 4 , and begin with the characteristic variables specified, i.e., (4.5). 
After Fourier transformation with respect to $y$, we obtain the differential-difference equations

$$
\left\{\begin{array}{l}
\partial \hat{U}_{j}(\omega) / \partial t+\hat{Q} \hat{U}_{j}(\omega)=0, \quad j=1, \ldots, N-1 \\
\hat{Q}=A_{+} D_{-x}+A_{-} D_{+x}+\hat{B} \\
\hat{\rho}_{0}(\omega)+\hat{u}_{0}(\omega)=0 \\
\hat{v}_{0}(\omega)=0 \\
\hat{\rho}_{N}(\omega)-\hat{u}_{N}(\omega)=0 .
\end{array}\right.
$$

It should be noted that the three boundary conditions given are sufficient to define $\hat{U}_{j}(\omega)$ at all inner points. When applying the scheme at $j=i$, only the combinations $\hat{p}+\hat{u}$ and $\hat{v}$ occur at $j=0$, and similarly only the combination $\hat{p}-\hat{u}$ occurs at $j=N$ when the scheme is applied at $j=N-1$. In order to obtain also the boundary values for all three variables, three extra boundary conditions must be defined, one at $j=0$ and two at $j=N$. Even if these conditions do not affect the inner solution, we state them here since they have been used also in combination with other methods. At $j=0$, the second equation of (5.1) is subtracted from the first to obtain a formula which does not include the point $j=-1$. Similarly, at $j=N$, the two first equations of (5.1) are added (to avoid the point $j=N+1$ ), and the equations for $v$ (the third component of $U$ ) can be used as they stand. We collect all the boundary conditions for the untransformed system:

Left boundary

$$
\left\{\begin{array}{l}
\rho_{0 k}+u_{0 k}=g_{k}^{\mathrm{I}}, \\
v_{0 k}=g_{k}^{\mathrm{II}}, \\
\frac{\partial}{\partial t}\left(\rho_{0 k}-u_{0 k}\right)+(1-c) D_{+x}\left(\rho_{0 k}-u_{0 k}\right) \\
\quad+\frac{c}{2} D_{-y}\left(\rho_{0 k}+v_{0 k}\right)-\frac{c}{2} D_{+y}\left(\rho_{0 k}-v_{0 k}\right)=0 ;
\end{array}\right.
$$

Right boundary

$$
\left\{\begin{array}{l}
\rho_{N k}-u_{N k}=g_{k}^{\mathrm{III}} \\
\frac{\partial}{\partial t}\left(\rho_{N k}+u_{N k}\right)+(1+c) D_{-x}\left(\rho_{N k}+u_{N k}\right) \\
\quad+\frac{c}{2} D_{-y}\left(\rho_{N k}+v_{N k}\right)-\frac{c}{2} D_{+y}\left(\rho_{N k}-v_{N k}\right)=0 \\
\frac{\partial}{\partial t} v_{N k}+D_{-x} v_{N k}+\frac{c}{2} D_{-y}\left(\rho_{N k}+v_{N k}\right)+\frac{c}{2} D_{+y}\left(\rho_{N k}-v_{N k}\right)=0
\end{array}\right.
$$

The spectrum for the operator $-\hat{Q}$ with the boundary conditions given in (5.2) were computed numerically, and the result is shown in Figure 5.2 for $\omega=1$.

The spectrum for the continuous operator is "bent down" towards the negative real axis when the differential operator is discretized; the picture is similar also for all the higher frequencies $\omega$. The consequence of this feature is that the use of upwind-differencing accelerates the convergence to steady state. We note, in particular, that the approximation of the lower part of the spectrum approaches the true 


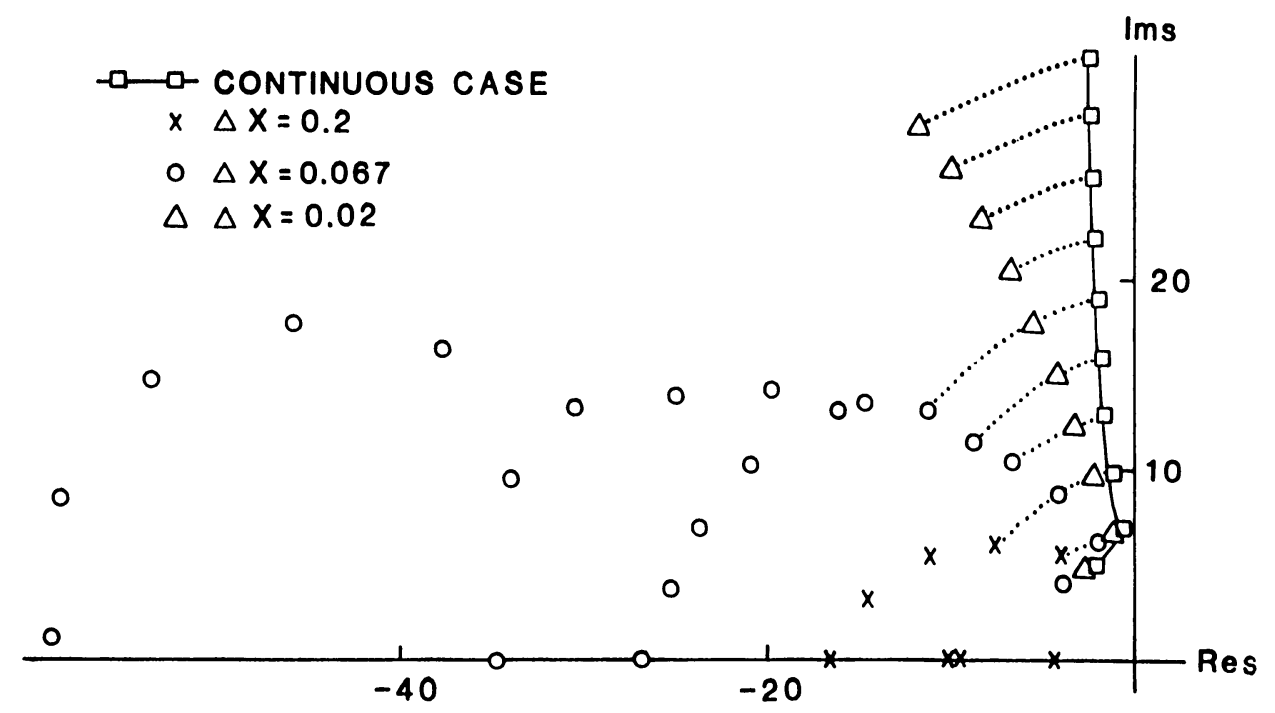

FIGURE 5.2

Spectrum of the upwind Fourier-transformed difference approximation to the subsonic Euler differential operator with characteristic boundary conditions. $\omega=1$. For the discrete cases, $\Delta x=\Delta y$. The dotted lines indicate the path of convergence as the step size decreases. (The upper half of the spectrum is displayed completely only for $\Delta x=0.2$ and $\Delta x=0.067$.)

values from the left, which is marked by dotted lines in the figure. (These eigenvalues have the smoothest eigenvectors, and they are therefore better approximated than the others when a coarse mesh is used for the difference operator.)

In practice, one often wants to avoid specifications of the particular combinations of variables corresponding to the characteristic variables at the boundaries. We have therefore investigated the case where the velocity and the density is specified according to (4.11). In Section 4 it was mentioned that the spectrum for the continuous operator is purely imaginary, which means that the solutions to the time-dependent problem do, in general, not converge at all. However, as shown in Figure 5.3, the spectrum for the upwind difference scheme is located in the left half-plane. This means that for every fixed grid the approximation will converge to a steady state which approximates the unique solution to the steady state continuous problem. One can observe from calculations with different number of mesh points $N$ that the largest real part of the spectrum to the discrete operator is well separated from zero, independent of $N$, for the highest frequency $\omega=N$. For $N=\omega=5$ we have the spectrum of the continuous operator in the set $\{s,|\operatorname{Im}(s)|>26, \operatorname{Re}(s)=0\}$ and the spectrum of the discrete operator in $\{s,|\operatorname{Im}(s)|<1, \operatorname{Re}(s)<-2\}$.

The spectral analysis above is based on the Fourier transform in the $y$-direction. It gives a relevant result regarding the asymptotic convergence rate in time. In order to study the convergence in real space explicitly, the original system of equations was 


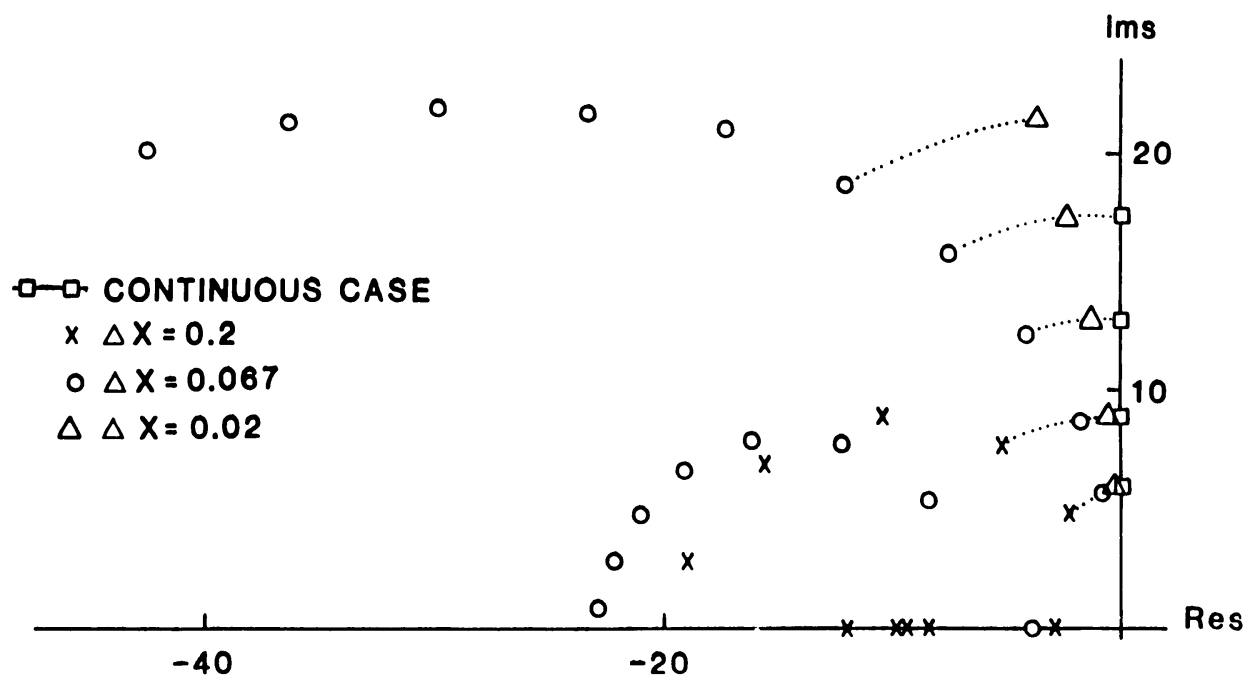

FIGURE 5.3

Spectrum of the upwind Fourier-transformed difference approximation to the subsonic Euler differential operator with specification of $u, v$ at $x=0$ and of $\rho$ at $x=1, \omega=1$, $\Delta x=\Delta y$. The dotted lines indicate the path of convergence as the step size decreases. (The upper half of the spectrum is displayed completely only for $\Delta x=0.2$.)

solved numerically. The two methods used were upwind differencing (UPW) in space, as described above, combined with the forward Euler difference method in time, and the Lax-Wendroff method (LW). For scalar equations, the latter method is identical to the method described in Section 3 with $c=\Delta t$ and with the forward Euler method in time. The Fourier expansions used in the analysis above are based on the assumption that $\rho$ and $u$ are symmetric across the boundaries $y=0,1$. For the limited computational domain, this corresponds to the conditions $\partial \rho / \partial y=$ $\partial u / \partial y=0$ at the boundaries. At the symmetry axis $y=0$ this is true, but at the solid wall $y=1$ it is, in general, not. For both difference methods we have therefore used boundary conditions that can be applied for general solutions. Since the coefficient matrices $B_{+}, B_{-}$have only zeros in the second row, the general approximation used at inner points for the second equation can be used also at the boundaries. By adding the first and third equation we get the missing condition at $y=1$. The total set of boundary conditions is composed by (5.3a), (5.3b) and the following conditions.

Lower boundary

$$
\left\{\begin{array}{l}
\rho_{j,-1}=\rho_{j 1}, \\
u_{j,-1}=u_{j 1}, \\
v_{j,-1}=v_{j 1} .
\end{array}\right.
$$


Upper boundary

$$
\left\{\begin{array}{l}
\frac{\partial}{\partial t} \rho_{j M}+\frac{1}{2}(1+c) D_{-x}\left(\rho_{j M}+u_{j M}\right)+\frac{1}{2}(1-c) D_{+x}\left(\rho_{j M}-u_{j M}\right) \\
\quad+c D_{-y}\left(\rho_{j M}+v_{j M}\right)=0 \\
\frac{\partial}{\partial t} u_{j M}+\frac{1}{2}(1+c) D_{-x}\left(\rho_{j M}+u_{j M}\right)+\frac{1}{2}(c-1) D_{+x}\left(\rho_{j M}-u_{j M}\right)=0 \\
v_{j M}=0
\end{array}\right.
$$

At the upper corners we use the special formulas (in order to avoid ambiguity and undefined variables):

Upper left corner

$$
\left\{\begin{array}{l}
\frac{\partial}{\partial t}\left(\rho_{0 M}-u_{0 M}\right)+(1-c) D_{+x}\left(\rho_{0 M}-u_{0 M}\right)+c D_{-y}\left(\rho_{0 M}+v_{0 M}\right)=0 \\
u_{0 M}+\rho_{0 M}=g_{M}^{\mathrm{I}} \\
v_{0 M}=g_{M}^{\mathrm{II}}
\end{array}\right.
$$

\section{Upper right corner}

$$
\left\{\begin{array}{l}
\frac{\partial}{\partial t}\left(\rho_{N M}+u_{N M}\right)+(1+c) D_{-x}\left(\rho_{N M}+u_{N M}\right)+c D_{-y}\left(\rho_{N M}+v_{N M}\right)=0 \\
\rho_{N M}-u_{N M}=g_{M}^{\mathrm{III}} \\
v_{N M}=0
\end{array}\right.
$$

All time-derivatives occurring in the boundary conditions are discretized by the forward Euler method. The boundary data are defined by

$$
\left\{\begin{array}{l}
g^{\text {I }}(y)=1-y^{2} \\
g^{\text {II }}(y)=0 \\
g^{\text {III }}(y)=0
\end{array}\right.
$$

and the initial data by

$$
\left\{\begin{array}{l}
\rho(x, y, 0)=1 \\
u(x, y, 0)=1-y^{2} \\
v(x, y, 0)=0
\end{array}\right.
$$

In order to simulate a real life problem, we have chosen the boundary data such that the steady state solution is not smooth. Putting all time derivatives equal to zero in the original system, we get from the third equation that $\partial \rho / \partial y=0$ at $y=1$ for all $x, 0 \leqslant x \leqslant 1$. The second equation shows, after differentiating with respect to $y$, that $\partial u / \partial y$ is constant along the upper boundary. Since $\partial(\rho+u) / \partial y=-2$ at $x=0, y=1$ we get $\partial u / \partial y=-2$ also at $x=1, y=1$. This implies that $\partial(\rho-u) / \partial y=2$ in the upper right corner, which does not match the data $\partial g^{\mathrm{III}} / \partial y=0$, and obviously there is a discontinuity in the first derivative of the solution. 
The convergence rate to steady state is shown in Figures 5.4-5.6 for different grid sizes. In all cases the two methods were run 500 time steps with $\Delta t=0.18 \Delta x$, which is close to the stability limit. This means that the final approximation $w^{500}$ corresponds to a smaller real time $t$ for a finer grid. The figures show the difference between $u^{n}$ and $u^{500}$ measured in the maximum norm, for $\Delta x=\Delta y=0.2,0.1,0.05$, respectively. The faster convergence for the upwind differencing method is quite obvious.

The upwind differencing method used here is only first-order accurate. By substituting the operator $D_{+}$by $D_{+}-\Delta x\left(D_{+}\right)^{2} / 2$ everywhere, we obtain secondorder accuracy in space. Another way of increasing the accuracy is to use Richardson extrapolation, which is also easy to apply for general conservation law problems.

Let $N=1 / \Delta x$ and $M=1 / \Delta y$ be even numbers and denote by $w^{(h)}(t)$ the approximation on the $N M$-grid. If $w^{(2 h)}(t)$ is the approximation for the $(N / 2) \times$ $(M / 2)$-grid, then the extrapolated second-order accurate approximation is

$$
\bar{w}_{j k}(t)=2 w_{2 j, 2 k}^{(h)}(t)-w_{j k}^{(2 h)}(t), \quad j=0,1, \ldots, N / 2, k=0,1, \ldots, M / 2 .
$$

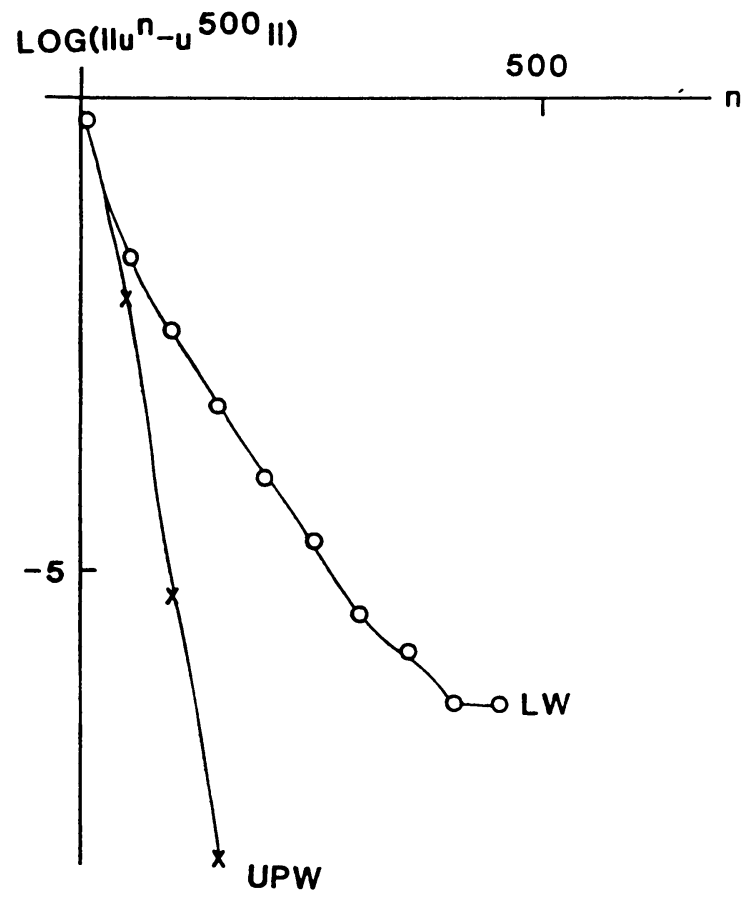

FIGURE 5.4

Convergence rate to steady state. The error as a function of the number of time steps $(n) . \Delta x=\Delta y=0.2$. LW-Lax-Wendroff scheme, UPW-upwind scheme. 


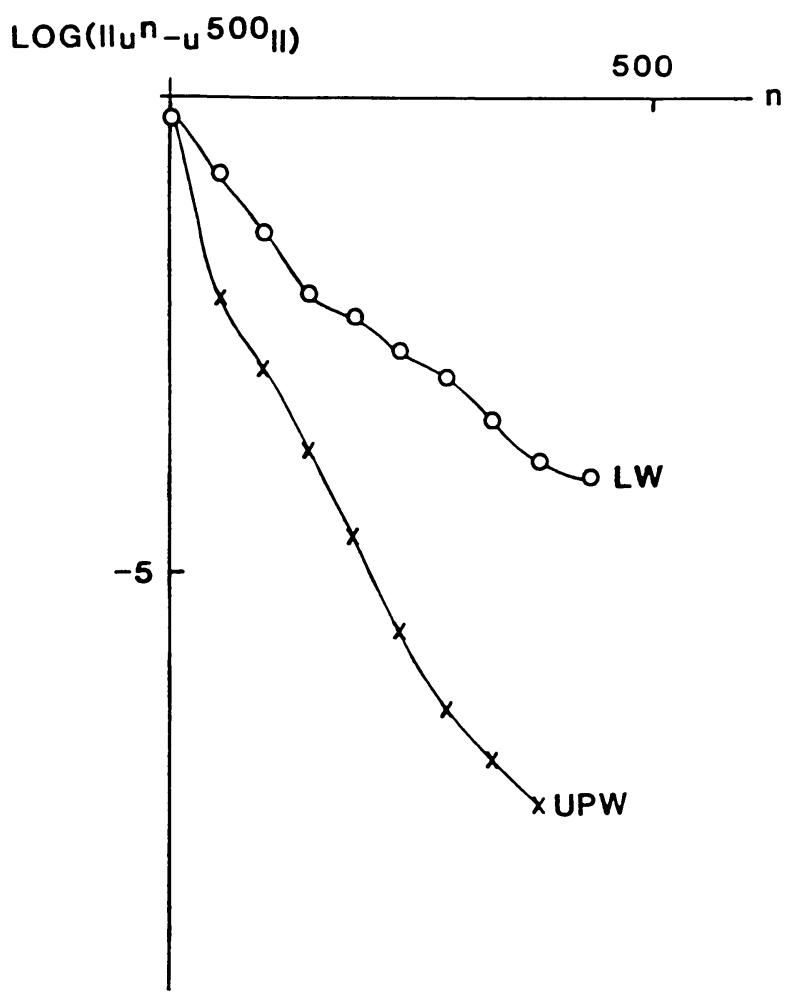

FIGURE 5.5

Convergence rate to steady state as in Figure 5.4 but with $\Delta x=\Delta y=0.1$.

We denote this approximation method by UPWR. To obtain the extrapolated steady state solution, the UPW-method was first run 500 steps on the $(N / 2) \times(M / 2)$-grid, which for our problem gave a converged solution $w^{(2 h)}(\infty)$. The function values were interpolated in space to provide the starting solution on the finer grid, and then another 500 steps were run, giving a converged solution $w^{(h)}(\infty)$. Finally, the extrapolation (5.6) was performed using these two solutions.

The error was computed for the three methods, using a converged approximation on a $40 \times 40$-grid as the exact solution. Because of the singularities in the corners, the error was measured in the max-norm over the inner square $0.2 \leqslant x, y \leqslant 0.8$ only. The result is shown in Table 5.1.

\section{TABLE 5.1}

The error after 500 steps

\begin{tabular}{r|ccc}
$N=M$ & UPW & LW & UPWR \\
\hline 5 & $3.910^{-2}$ & $2.410^{-2}$ & \\
10 & $2.010^{-2}$ & $4.910^{-3}$ & $4.410^{-3}$ \\
20 & $1.010^{-2}$ & $2.810^{-3}$ & $1.210^{-3}$
\end{tabular}




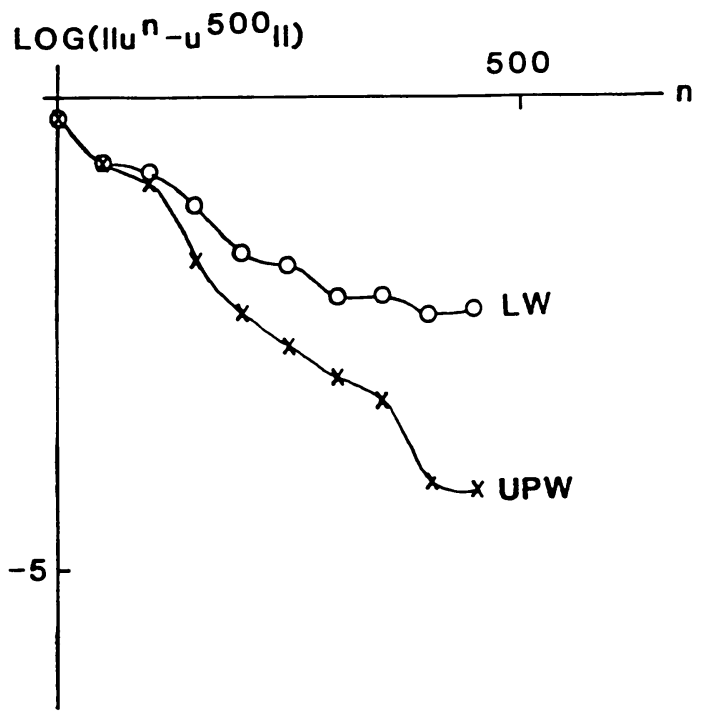

FIGURE 5.6

Convergence rate to steady state as in Figure 5.4 but with $\Delta x=\Delta y=0.05$.

The Lax-Wendroff method exhibits a poor performance on the finest grid, and this is due to the slow convergence in time (see Figure 5.6). We can also see that the iterations could have been stopped much earlier for the UPW-scheme. For example, if we are interested in a precision of the order $3 \cdot 10^{-3}$ only (which is obtained with the LW-scheme), then the UPW-scheme or the finest grid could have been stopped after 200 steps. Since the number of operations per step is smaller for upwind-differencing, the gain in efficiency is larger than 2.5 .

Department of Mathematics

University of California

Los Angeles, California 90024

Department of Computer Sciences

Uppsala University and

National Defense Research Institute (FOA)

Stockholm, Sweden

1. B. ENGQUist \& S. OShER, "Stable and entropy satisfying approximations for transonic flow calculations," Math. Comp., v. 34, 1980, pp. 45-75.

2. L. E. ERIKSSON \& A. Rizzı, "Computer-aided analysis of the convergence to steady state of discrete approximations to the Euler equations," J. Comput. Phys., v. 57, 1985, pp. 90-128.

3. L. FERM \& B. GUSTAFSSON, "A down-stream boundary procedure for the Euler equations," Comput. \& Fluids, v. 10, 1982, pp. 261-276.

4. M. B. GILES, Eigenmode Analysis of Unsteady One-Dimensional Euler Equations, ICASE Report No. $83-47,1983$.

5. S. K. Godunov, "A difference method for numerical calculation of discontinuous solutions of the equation of hydrodynamics," Mat. Sh., v. 47, 1959, pp. 271-306.

6. H.- O. Kreiss, "Initial boundary value problems for hyperbolic systems," Comm. Pure Appl. Math., v. 23, 1970, pp. 277-298. 
7. C. S. Morawetz, "The decay of solutions of the exterior initial-boundary value problem for the wave equation," Comm. Pure Appl. Math., v. 14, 1961, pp. 561-568.

8. C. S. Morawetz, "Decay of solutions of the exterior problem for the wave equation," Comm. Pure Appl. Math., v. 28, 1975, pp. 229-264.

9. C. S. Morawetz, J. V. Ralston \& W. A. Strauss, "Decay of solutions of the wave equation outside nontrapping obstacles," Comm. Pure Appl. Math., v. 30, 1977, pp. 447-508.

10. J. Oliger \& A. SUNDSTRÖM, “Theoretical and practical aspects of some initial boundary value problems in fluid dynamics," SIAM J. Appl. Math., v. 35, 1978, pp. 419-446.

11. S. OSHER \& F. SOlOMON, "Upwind difference schemes for hyperbolic systems of conservation laws," Math. Comp., v. 38, 1982, pp. 339-374.

12. P. L. RoE, “Approximate Riemann solvers, parameter vectors, and difference schemes," J. Comput. Phys., v. 43, 1981, pp. 357-372.

13. E. Turkel, Progress in Computational Physics, ICASE report No. 82-83, 1982.

14. E. TURKEl, Acceleration to a Steady State for the Euler Equations, ICASE Report No. 84-32, 1984. 